CASE REPORT

\title{
Video illustration of staple gun use to rapidly repair on-field head laceration
}

\section{J W Orchard}

Br J Sports Med 2004;38:e7 (http://www.bjsportmed.com/cgi/content/full/38/4/e7). doi: 10.1136/bjsm.2003.005470

\begin{abstract}
A professional rugby league player sustained a left eyebrow laceration during a match which immediately started to bleed. Within seconds he was taken to the sideline and had the laceration closed with six staples by the team doctor. Bleeding was arrested and he returned to play, taking the ball within $80 \mathrm{~s}$ of suffering the initial laceration, and within $40 \mathrm{~s}$ of the stapling procedure. This sequence was captured on video and appeared on television. The staples were removed after the match and the wound sutured. Repair of the wound was uneventful. The staple gun allows bleeding lacerations to be closed within seconds and for players to safely and quickly return to play, whilst minimising the risk of blood-borne infection transmission.
\end{abstract}

$\mathrm{H}$ ead and facial lacerations are among the most common injuries in contact sports where headgear is not generally used, such as rugby league, rugby union, Australian football, and professional boxing. In the past, they were considered to be injuries of very little consequence. Over the last decade, concern about transmission of blood-borne viruses has meant that most sports have enacted rules that prevent athletes from remaining on the field of play when they are actively bleeding. The risks of on-field transmission of blood-borne viruses have been described as "negligible" but remotely possibly. ${ }^{1}$ Because professional sport needs to be continuous, the rules usually allow play to continue while the bleeding player is off the field. Therefore, rapid closure of open wounds is an important aim for doctors treating athletes in collision sports, in order to minimise the time that players are unavailable for play whilst also minimising the risk of transmission of blood-borne infections.

This case involved the full-back for the Sydney Roosters, an Australian professional team in the National Rugby League (NRL). He took the ball on a kick return at the 8:38 min mark of the game and was tackled and immediately suffered a bleeding laceration of the forehead just above the left eyebrow. As is often the case, the referee did not immediately notice that the player had suffered an open facial laceration and play continued. The player was soon attended by a team trainer (8:53) who recommended the player leave the field. He moved quickly over to the sideline (9:06) and had the wound closed by the author (9:20-9:25), who was the team's doctor, using six staples from a disposable Leukoclip SD staple gun (Beiersdorf, Hamburg) (fig 1). The wound ceased bleeding immediately and after blood was wiped from the player's face, he returned to play. He then was able to again field a ball from the next kick return at the 9:57 min mark of the game. This procedure and sequence were captured by television cameras and broadcast live on Channel Nine (Sydney) "Friday Night Football".

Following the end of the game, the player was definitively treated in the dressing room under local anaesthetic block.

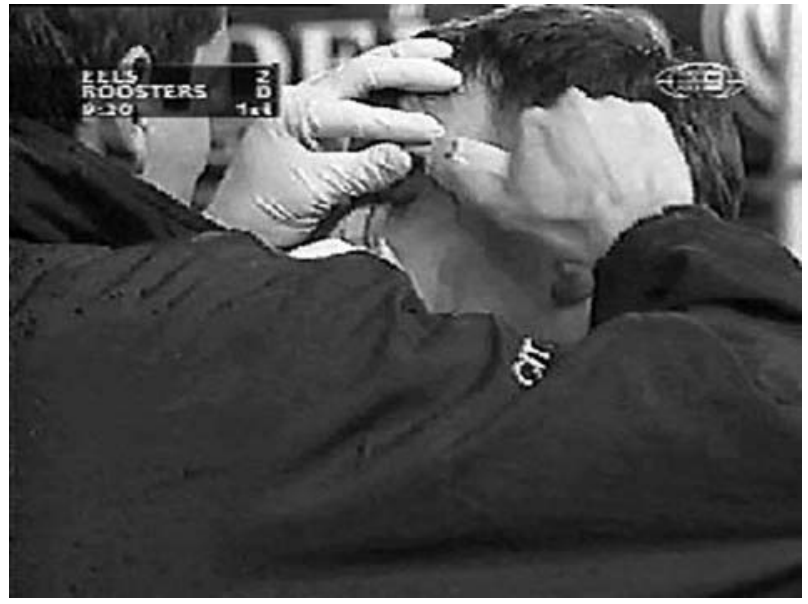

Figure 1 Still frame from the video.

He had the staples removed, and the wound cleaned and then repaired with six 5/0 Dafilon sutures (Braun, Tuttlingen). The wound healed uneventfully, with the sutures removed a week later.

\section{DISCUSSION}

Staples have been used as a common method for closure of certain wounds in operating theatres and emergency departments for 15 years. Their use in this particular case was controversial and discussed in detail by the Sydney media, with the point of controversy being whether this incident should have appeared on television. Although the author has used the staple gun to rapidly repair 70 wounds (without any significant complications) in professional football players over the last 10 years, this was the first occasion that the procedure was captured by television cameras and discussed by media commentators.

The NRL administration felt that live stapling procedures did not present a good "image" of the game, and made the recommendation that this procedure not be carried out near television cameras for aesthetic (rather than medical) reasons. There was a mixed reaction by the media to this decision. For example, a Sydney journalist who watched the game wrote: "If you'll pardon the pun, this was riveting stuff. Moments after being rushed back on to the field with his head stapled up, Phillips was fearlessly hurling himself at the opposition. Channel 9's Ray Warren marvelled at how courageous Phillips was. How, within $90 \mathrm{~s}$ of having sustained the gash by throwing himself at Parramatta players, there he was back out there doing the very same thing. For me, it was a graphic insight into the reality of how tough our rugby league players are ${ }^{\prime 2}{ }^{2}$ Since the initial event, a

Abbreviations: NRL, National Rugby League 


\section{Take home message}

The staple gun can be used to rapidly repair certain scalp and facial lacerations in professional football players within seconds. It is the most efficient method for rapidly closing wounds. In the heat of the game, the pain of receiving staples without local anaesthetic is minimal to a professional footballer. Staples can be removed at the completion of the game and the wound definitively treated with sutures, which are the optimum form of management on the face but which require players to be absent from the game for a lengthy period.

very similar case occurred during a rugby league 2003 State of Origin match, re-igniting the debate about whether these incidents should be shown on television. As with the initial case, there were diametrically opposed and passionately argued opinions that the filming of such a procedure was either an exciting addition to the television coverage or was barbaric and had the potential to turn viewers off the sport.

The issue of whether or not the images of stapling procedures are acceptable television is different to whether this form of management is an acceptable way to manage head lacerations in professional football players. The author first reported this technique 10 years ago in Sport Health, an Australian sports medicine magazine. ${ }^{3}$ A letter to the editor in the following issue outlined a case why staples should not be used on the field to repair facial lacerations, including cosmetic reasons, lack of sterility, and risk of further damage. ${ }^{4}$ Neither of these objections has led to any compromised outcomes (for example, infections, poor cosmetic outcomes, damage to other players) in this author's subsequent personal series of 70 cases. The argument that on-field procedures are not performed in a sterile environment is somewhat countered by the speed of closure, which minimises the time that pathogens can enter an open wound. Wounds can be cleaned after the game during definitive treatment with sutures, ensuring a good cosmetic result for simple wounds. Obviously not all wounds are suitable for stapling, including those involving the eyelid, lips, nose, and ears. Staples are a thicker foreign body than regular sutures and hence there is a theoretical risk that they are more likely to injure an opponent if there is a further collision. This is unlikely as the staples do not provide any sharp edges and protrude only a millimetre beyond the normal contour of the skin. This theoretical complication can be eliminated if the staples are covered by an additional bandage. The strength of staples also helps in preventing the wound from re-opening if there is a further collision, which not uncommonly occurs with regular fine sutures.

The case in favour of using staples is simple, with the enormous advantage being the speed of the procedure. This has an obvious performance advantage for the player and the team as players are unavailable for a far shorter times. In the real world, it is likely that the use of staples minimises the infection risk to other players. Although the various rules of rugby league and similar sports generally require a bleeding player to immediately be removed from the field for definitive wound closure, the reality is that this does not always happen. This is particularly the case in the NRL, where teams are restricted to 12 interchanges per match (including those involving a bleeding player). Players and their teams place a priority on performance, which often means continuing with play whilst bleeding so as not to leave the team a man "short", over the infinitely small risk that they may transmit a blood-borne infection to another player. Rather than remove a player from the field or take him away from play, team trainers in the NRL will often wrap an openly bleeding wound with gauze and tape, which then slowly leaks blood over the remainder of the game or half, constituting a greater infection risk than if the wound had been immediately closed with staples. A consequence of the limited interchange rule is that referees usually give a player with minor bleeding some leeway before they insist on his removal from the field (which penalises the team as they must either play a man "short" or forfeit an interchange in replacing him). The NRL and other rugby league competitions have in the past allowed teams a "free" interchange for bleeding players, which was discontinued because of abuse in the other direction (that is, team trainers deliberately tried to aggravate minor bleeding wounds on tired players in the hope that the referee would stop play and force a "free" substitute). This history of abuse of the "blood bin" is a good illustration of how "optimum" medical management is often considered secondary to team performance in a fully professional environment.

This case study does not offer a complete solution to the difficult dilemma that professional sports face with respect to maintaining a continuous game but reducing the risk of transmission of blood-borne infections. It does illustrate an efficient technique which can help to minimise the problem of players being absent from continuous play due to bleeding from open wounds on the field.

\section{ELECTRONIC-DATABASE INFORMATION}

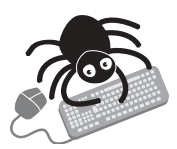

A video of the incident is available at http:// www.nrl.com.au/multimedia/ telstravideo.cfm? $\mathrm{S}=2 \& \mathrm{R}=41 \& \mathrm{~T}=($ select the Eels $v$ Roosters game). There is also a link to the video at http://www.injuryupdate.com.au/injuries/head_\& neck/facial_lacerations.php? menu = injury_details\& menu2 =head_and_neck )

Correspondence to: John W Orchard, South Sydney Sports Medicine, 111 Anzac Parade, Kensington, NSW 2033, Australia;

johnorchard@msn.com.au

Received 23 July 2003

Accepted for publication 28 July 2003

\section{REFERENCES}

1 Dorman JM. Contagious diseases in competitive sport: what are the risks? $J$ Am Coll Health 2000:49:105-9.

2 Gibson M. Ultimate in reality TV. Daily Telegraph, (Sydney) 2002; June 5.

3 Orchard J. Wound management in football. Sport Health 1994;12(3):23-4.

4 Garnham A. Letter to the editor. Sport Health 1994; 12(4):5-6. 